\title{
Enhanced Human Decidual Cell-Expressed FKBP51 May Promote Labor-Related Functional Progesterone Withdrawal
}

Frederick Schatz, ${ }^{*}$ Ozlem Guzeloglu-Kayisli, ${ }^{*}$ Murat Basar, ${ }^{\dagger}$ Lynn F. Buchwalder, ${ }^{\dagger}$ Nehir Ocak, ${ }^{\dagger}$ Elif Guzel, ${ }^{\dagger \ddagger}$ Seth Guller, ${ }^{\dagger}$ Nihan Semerci, ${ }^{*}$ Umit A. Kayisli, ${ }^{*}$ and Charles J. Lockwood*

From the Department of Obstetrics and Gynecology, * University of South Florida, Morsani College of Medicine, Tampa, Florida; the Department of Obstetrics, Gynecology, and Reproductive Sciences, ${ }^{\dagger}$ Yale University School of Medicine, New Haven, Connecticut; and the Department of Histology and Embryology, ${ }^{\ddagger}$ Istanbul University, Cerrahpasa Medical Faculty, Istanbul, Turkey

Accepted for publication

May 21, 2015.

Address correspondence to Charles J. Lockwood, M.D., University of South Florida, Morsani College of Medicine, 12901 Bruce B. Downs Blvd, Tampa, FL 33612. E-mail: cjlockwood@health.usf.edu.
Sustained plasma progesterone (P4) levels suggest initiation of human term labor by functional P4 withdrawal, reflecting reduced progesterone receptor (PR) and/or glucocorticoid receptor (GR) expression or activity. The steroid-induced immunophilin cochaperone FKBP51 inhibits PR- and GR-mediated transcription, suggesting a labor-initiating role. Gestational age-matched decidual sections were immunostained for FKBP51 and decidual cell (DC) and interstitial trophoblast (IT) markers, vimentin and cytokeratin, respectively. Term DC cultures were incubated with vehicle (control), estradiol $\left(E_{2}\right)$ with or without medroxyprogesterone acetate, dexamethasone (Dex), or Organon 2058. FKBP51 histologic scoring was significantly higher in DC nuclei during labor versus prelabor decidua, whereas FKBP51 immunostaining was undetected in interstitial trophoblasts $(P<0.05)$. In term $D C$ cultures, $\mathrm{E}_{2}+$ medroxyprogesterone acetate or $\mathrm{E}_{2}+$ Dex enhanced FKBP51 expression $(P<0.01)$, whereas $\mathrm{E}_{2}+$ Organon 2058 inhibited PR expression $(P<0.05)$, and $\mathrm{E}_{2}+$ Dex inhibited GR expression $(P<0.05)$. Unlike term DCs, FKBP51 was undetected in control or Dex-treated cultured third-trimester trophoblasts. Electrophoretic mobility shift assays revealed that FKPB51 overexpression or silencing in cultured DCs altered PR-DNA binding. Increased FKBP51 levels in term DCs during labor complement our prior in situ observations of significantly lower PR in labor versus prelabor DCs. In a milieu of sustained plasma P4 levels, these reciprocal changes will amplify functional P4 withdrawal in DCs via FKBP51-mediated PR resistance coupled with declining PR levels, whereas the lack of FKBP51 expression in interstitial trophoblasts suggests unopposed constitutive GR action. (Am J Pathol 2015, 185: 2402-2411; http://dx.doi.org/10.1016/j.ajpath.2015.05.014)
Progesterone receptor (PR) isoforms, PR-A $(92 \mathrm{kDa})$ and PR-B (116 kDa), bind progesterone (P4) with equal affinity and are encoded by a single gene controlled by distinct estrogen-sensitive promoters. ${ }^{1}$ In most mammals, high endogenous P4 levels maintain myometrial quiescence, ${ }^{2}$ and declining plasma P4 levels trigger labor. ${ }^{3}$ However, in humans, plasma P4 levels remain elevated during labor. ${ }^{4,5}$ That P4 withdrawal can mediate human labor is suggested by the induction of cervical ripening and labor after administration of the PR antagonist mifepristone (RU486) or inhibitors of $3 \beta$-hydroxysteroid dehydrogenase, which lower systemic P4 levels. ${ }^{7}$ Moreover, human labor is associated with functional P4 withdrawal mediated by lower myometrial PR levels and/or a shift to dysfunctional PR isoform dominance. ${ }^{2,8,9}$

Previously, we compared PR immunostaining in gestational age $(\mathrm{GA})$-matched prelabor and post-labor decidua basalis and parietalis ${ }^{10}$ and detected nuclear PR in decidual cells (DCs), but not in adjacent interstitial trophoblasts (ITs). The presence of significantly lower PR levels in the DCs of post-labor versus prelabor basalis and parietalis is consistent with functional P4 withdrawal. ${ }^{10}$ The observations that

Supported by the March of Dimes Foundation Ohio Prematurity Collaborative grant 22-FY14-470 (C.J.L.).

Disclosures: None declared. 
enhanced levels of proparturition, placental-derived corticotrophin-releasing hormone result from diminished competition between stable $\mathrm{P} 4$ and increasing maternal and/or fetal cortisol levels for glucocorticoid receptor (GR) binding ${ }^{11}$ and reports that mifepristone (RU486) is both a PR and GR antagonist $^{12,13}$ prompted GR immunostaining in decidual sections. ${ }^{10}$ In contrast with PR immunostaining in DCs but not ITs, immunoreactive GR was detected at lower levels in DC nuclei than in ITs. Moreover, unlike the labor-associated decreases in PR levels seen in DCs, neither DCs nor ITs displayed labor-associated changes in GR levels. ${ }^{10}$

The FK506-binding proteins, FKBP51 and FKBP52, are immunophilin binding proteins that assemble as cochaperones with heat shock protein 90 at the ligand-binding domain of PR-A, PR-B, ${ }^{14}$ and GR. ${ }^{15,16}$ FKBP52 enhances and FKBP51 attenuates PR- and GR-mediated transcription. ${ }^{15,16}$ Potential competition between FKBP51 and FKBP52 at the ligandbinding domain suggests that each heat shock protein 90 cochaperone indirectly affects the activity of the other. ${ }^{17}$ Preceding steroid binding, PR and GR assemble sequentially with chaperones. During the final assembly stage, heat shock protein 90 associates with the ligand-binding domain to stabilize both PR and GR ligand binding. Hormone binding completes the PR and GR chaperone assembly cycle to activate each steroid receptor as a transcription factor. ${ }^{14,17}$ In DCs, the FKBP51 gene, but not the FKBP52 gene, is under robust up-regulation by respective steroid hormones rendering FKBP51 protein as a component of an ultrashort negative feedback loop that regulates steroid signaling ${ }^{18}$ and may mediate functional $\mathrm{P} 4$ withdrawal.

We evaluated the potential role of FKBP51 in mediating functional P4 withdrawal by studying the following: i) laborrelated changes in FKBP51 expression in DCs and ITs in decidua basalis and parietalis sections; ii) regulation of FKBP51 and FKBP52, as well as PR and GR expression, in cultured human term DCs incubated with vehicle (control, C) or estradiol $\left(E_{2}\right)$ alone, or with Organon 2058 (Org2058), a pure progestin, dexamethasone (Dex), a pure glucocorticoid, or medroxyprogesterone acetate (MPA), a mixed progestinglucocorticoid $^{19,20}$; and iii) function of FKBP51 on PR binding to its canonical progesterone response element (PRE) in FKBP51-overexpressed or silenced term DCs.

\section{Materials and Methods}

\section{Tissues}

After receiving informed consent, placentas were obtained at the Yale-New Haven Hospital (New Haven, CT) under Yale University Human Investigation Committee approval. Biopsy specimens from decidua basalis and parietalis were obtained before evidence of regular contractions on tocodynamometer at the time of a repeat cesarean delivery $(n=8)$ and after onset of spontaneous labor with regular contractions resulting in vaginal delivery $(n=9)$. For prelabor and post-labor cases (means \pm SD), maternal ages were $30.6 \pm 5.6$ and $28.0 \pm 5.8$ years, respectively; and GAs were $39.1 \pm 0.5$ and $39.1 \pm 1.2$ weeks, respectively $(P>0.05)$. Patients displayed no evidence of abruption (ie, vaginal bleeding or hypofibrinogenemia) or either histological or clinical chorioamnionitis. ${ }^{21}$

\section{Isolation and Culture}

Term DCs were isolated, as previously described, ${ }^{22,23}$ from uncomplicated pregnancies undergoing repeat cesarean delivery at term under Yale Human Investigation Committee approval $(n=7)$. Briefly, after removal of the amnion from placental membranes, parietalis was scraped from the chorion, whereas basalis was scraped from the placental surface distal to the intervillous space. The tissues were each digested by collagenase-deoxyribonuclease, purified on a Percoll gradient, grown to confluence, and passaged until found to be $>99 \%$ free of $\mathrm{CD} 45^{+}$cells by flow cytometry. ${ }^{22}$ Confluent term DCs were primed in serum-containing medium with vehicle, $10^{-8} \mathrm{~mol} / \mathrm{L}$ $\mathrm{E}_{2}, 10^{-7} \mathrm{~mol} / \mathrm{L} \mathrm{MPA}, 10^{-7} \mathrm{~mol} / \mathrm{L}$ Dex (Sigma-Aldrich, St. Louis, MO), $10^{-7} \mathrm{~mol} / \mathrm{L}$ Org2058 (Organon Pharmaceuticals USA Inc., currently under the name of Merck \& Co, Whitehouse Station, NJ), $E_{2}+$ MPA, $E_{2}+$ Dex, or $E_{2}+$ Org2058 for 7 days, then switched to defined medium with corresponding steroids for 24 hours, as described previously. ${ }^{23}$ Term DCs were scraped into ice-cold extraction buffer with protease inhibitors, and cytoplasmic and nuclear extracts were isolated (Thermo Scientific, Rockford, IL). Total RNA was extracted from parallel 6-hour incubations.

\section{CYT and SCT Isolation and Culture}

Isolation of cytotrophoblasts (CYTs) from placentas was performed as described. ${ }^{24}$ Placentas were obtained after uncomplicated pregnancies and repeat cesarean deliveries at term using procedures approved by the Yale Human Investigation Committee. Briefly, villous tissue was initially digested with trypsin/deoxyribonuclease $\mathrm{I}$, and centrifuged on a discontinuous Percoll gradient (50\%/45\%/35\%/30\%). Cells migrating to the $35 \% / 45 \%$ Percoll interface were recovered by centrifugation and were immunopurified using negative selection by simultaneous treatment with mouse anti-human CD9 antibody, mouse anti-human CD45 antibody, and then goat anti-mouse IgG-conjugated DynaBeads (Life Technologies, Grand Island, NY), yielding CYTs with $>98 \%$ purity on the basis of immunocytochemical and flow cytometric analysis. The decidua basalis layer is trimmed off before digestion, leaving primarily villous tissue for CYT isolation. ${ }^{24}$ SCTs were obtained by spontaneous differentiation of CYTs after 72 hours of culture, as previously described. ${ }^{25}$ CYTs and SCTs were treated with $10^{-7} \mathrm{~mol} / \mathrm{L}$ Dex for 6 hours, and RNA was extracted for further studies.

\section{Immunohistochemistry and HSCORE Analysis}

Triple immunostaining was performed in formalin-fixed, paraffin-embedded sections with antigen retrieval using 
citrate buffer, as described previously. ${ }^{10}$ Sections were incubated overnight with a goat polyclonal antibody against FKBP51 (R\&D, Minneapolis, MN), diluted $1: 2000$, then with a mouse monoclonal antibody against cytokeratin 7 (Dako, Carpinteria, CA), and finally with a chicken polyclonal antibody against vimentin (Abcam, Cambridge, MA). Slides were incubated with biotinylated secondary antibodies (diluted 1:1000; Jackson Labs, West Grove, PA), followed by incubation with streptavidinconjugated peroxidase or alkaline phosphatase (Vector Labs, Burlingame, CA). Chromogens that were subsequently used to visualize immunoreactivity included 3,3diaminobenzidine for FKBP51 (brown), Vector SG for cytokeratin (gray), and Vector RED for vimentin (Vector Labs). FKBP51immunoreactivity was semiquantitatively evaluated using histological score (HSCORE) analysis, as described. ${ }^{10}$ HSCOREs were evaluated at different times by two observers (E.G., N.O.) blinded to tissue type and clinical outcome. The average scores of both were used as the final HSCORE for each sample. The intraindividual and interindividual coefficients of variation were $10 \%$ and $13 \%$, respectively.

\section{Real-Time Quantitative PCR}

Total RNA from term DCs and trophoblasts was isolated and purified using a RNAqueous-Micro isolation kit, according to the manufacturer's instructions (Ambion, Austin, TX). Reverse transcription was performed using the $\mathrm{Su}$ perScript II kit (Invitrogen, Carlsbad, CA) in two steps: template RNA and random primers were incubated at $65^{\circ} \mathrm{C}$ for 5 minutes to eliminate any secondary structures, and then the buffer and enzyme were added and the reaction was performed at $42^{\circ} \mathrm{C}$ for 1 hour. Reverse transcriptase was inactivated at $70^{\circ} \mathrm{C}$ for 10 minutes. Real-time quantitative PCR (qPCR) was performed with TaqMan gene expression assays for FKBP51, FKBP52, vimentin, IL-1 $\beta$, and the reference gene, $\beta$-actin (Applied Biosystems, Foster City, CA). Standard curves were generated from serial dilutions of a known sample for each assay. All samples were run in triplicate, and the average was used for each sample. The $2^{-\Delta \Delta C_{T}}$ (cycle threshold) method was used to calculate relative expression levels. Results were reported as a fold change (Table 1).

\section{Immunoblotting Analyses}

Nuclear and cytoplasmic extracts from term DCs $(50 \mu \mathrm{g}$ per lane) and positive control T47D cells ( $8 \mu \mathrm{g}$; Abcam) were subjected to reducing SDS-PAGE on a $10 \%$ Tris- $\mathrm{HCl}$ gel (Bio-Rad, Hercules, CA). Transfer onto nitrocellulose, blocking, chemiluminescence, and stripping were performed as described. ${ }^{23}$ Immunoblotting used a goat polyclonal, for FKBP51, a mouse monoclonal antibody specific for human PR, GR, and a rat monoclonal antibody for FKBP52 (R\&D), followed by incubation with horseradish peroxidaseconjugated rabbit anti-goat, goat anti-mouse, or donkey anti-rat
Table 1 Detailed Description of Primers Used for Real-Time Quantitative PCR

\begin{tabular}{ll}
\hline Primer/probe name & TaqMan assay ID \\
\hline FKBP51 & Hs01561006_m1 \\
FKBP52 & Hs00427038_g1 \\
$\beta$-Actin & Hs99999903_m1 \\
Vimentin & Hs00185584_m1 \\
IL-1 $\beta$ & Hs0155410_m1 \\
\hline
\end{tabular}

antibodies (Jackson Labs). Immunoblotting results were normalized by stripping membranes and reprobing with a TATA-binding protein (TBP) monoclonal antibody (Abcam) for nuclear extracts, or with a $\beta$-actin monoclonal antibody (Cell Signaling, Danvers, MA) for cytosolic extracts. Band intensities were quantified using computer densitometry analysis (ImageJ software version 1.49; NIH, Bethesda, MD) ${ }^{26}$ (Table 2).

\section{FKBP51 and FKBP52 0verexpression}

To overexpress FKBP51 and FKBP52 in term DCs, expression vector containing the full-length FKBP51 gene open reading frame (pCMV6-FKBP51 or pCMV6FKBP52) and empty vector (pCMV6) were purchased from Origene Inc. (Rockville, MD). Transient transfections were performed in term DCs (approximately 70\% confluence) using Lipofectamine LTX reagent (Invitrogen, Carlsbad, CA) in Opti-MEM I serum-reduced medium (Invitrogen), according to the manufacturer's instructions. After 44 hours of transfection, DCs transfected with FKBP51 were incubated with $E_{2}$ alone or $E_{2}+\operatorname{Org} 2058$ for 4 hours, then washed with ice-cold phosphate-buffered saline twice and stored in $-80^{\circ} \mathrm{C}$ for subsequent experiments. Cell extracts from DC cultures transiently transfected with control versus FKBP52 expression vectors were used to confirm the high specificity of the FKBP52 antibody (Supplemental Figure S1).

Table 2 Detailed Description of Antibodies Used for Immunohistochemistry and Immunoblotting

\begin{tabular}{|c|c|c|c|}
\hline Antibody name & Species & $\begin{array}{l}\text { Dilution } \\
\text { used }\end{array}$ & $\begin{array}{l}\text { Catalog no. } \\
\text { (company) }\end{array}$ \\
\hline $\begin{array}{l}\text { Progesterone } \\
\text { receptor }\end{array}$ & Mouse & $1: 200$ & $\begin{array}{l}\text { M3568 (Dako, } \\
\text { Carpinteria, CA) }\end{array}$ \\
\hline $\begin{array}{l}\text { Glucocorticoid } \\
\text { receptor }\end{array}$ & Mouse & $1: 500$ & $\begin{array}{l}\text { PP-H80004 (R\&D, } \\
\text { Minneapolis, MN) }\end{array}$ \\
\hline FKBP51 & Goat & $1: 2000$ & AF4094 (R\&D) \\
\hline FKBP52 & Rat & $1: 500$ & MAB4095 (R\&D) \\
\hline$\beta$-Actin & Rabbit & $1: 5000$ & $\begin{array}{l}51,255 \text { (Cell Signaling, } \\
\text { Danvers, MA) }\end{array}$ \\
\hline TATA-binding protein & Mouse & 1:1500 & $\begin{array}{l}\text { T1827 (Sigma-Aldrich, } \\
\text { St. Louis, M0) }\end{array}$ \\
\hline Cytokeratin & Mouse & $1: 600$ & M7018 (Dako) \\
\hline Vimentin & Chicken & $1: 300$ & $\begin{array}{c}\text { Ab-39376 (Abcam, } \\
\text { Cambridge, MA) }\end{array}$ \\
\hline
\end{tabular}




\section{Silencing of FKBP51 by siRNA-Mediated Knockdown}

Term DCs were transfected with either FKBP51-specific siRNA or non-specific siRNA (Invitrogen) as a control using Lipofectamine RNAiMax Reagent, according to the manufacturer's instructions (Invitrogen). Forty-four hours after transfection, cells were incubated with $\mathrm{E}_{2}$ or $\mathrm{E}_{2}+$ Org2058 for 4 hours, then washed with ice-cold phosphatebuffered saline twice, and stored in $-80^{\circ} \mathrm{C}$ for subsequent nuclear protein extraction or RNA isolation. Transfection efficiency for either overexpression or silencing of FKBP51 was confirmed by qPCR and immunoblotting analyses (data not shown).

\section{EMSA Data}

Electrophoretic mobility shift assay (EMSA) was performed as described previously, with a slight modification. ${ }^{27}$ After experimental DC incubations, nuclear extracts were isolated according to the manufacturer's instruction (Active Motif, Carlsbad, CA), and protein was quantified by the Bradford method. ${ }^{28}$ The PR EMSA kit includes biotin-labeled and unlabeled PR consensus binding site oligonucleotides (PRE; Signosis Inc., Santa Clara, CA). Nuclear extracts $(2 \mu \mathrm{g}$ per sample) were incubated with PRE-oligonucleotides in binding buffer and stabilization solution for 30 minutes at $22^{\circ} \mathrm{C}$. A mutant PRE probe and a 100 -fold excess of unlabeled PRE probe were used in PR binding control and competition assays, respectively. All samples were loaded onto nondenaturing $6.5 \%$ polyacrylamide gel wells in $0.5 \times$ Tris-boric acid-EDTA buffer at $4^{\circ} \mathrm{C}$, followed by transfer to membrane. The membrane was cross-linked by UV-linker (Stratagene, La Jolla, CA) and incubated with HRP-linked streptavidin at room temperature for 1 hour, and the signal was then developed by enhanced chemiluminescence chemiluminescence reagents (Thermo Fisher Scientific, Rockford, IL).

\section{Statistical Analysis}

qPCR results for FKBP51 and FKBP52, immunoblot results for FKBP51, FKBP52, PR, and GR, and HSCORE results for FKBP51 were normally distributed and analyzed by oneway analysis of variance with a post hoc Holm-Šidák test. Statistical calculations were performed using SigmaStat version 3.0 (Systat Software, San Jose, CA).

\section{Results}

Consecutive triple immunostaining (Figure 1) was performed initially with an FKBP51-specific primary antibody, followed by vimentin- and cytokeratin-specific antibodies. In all sections, FKBP51 was detected exclusively in DC nuclei but was absent in ITs. Relative FKBP51 immunoreactivity was evaluated in decidua basalis and parietalis from women not experiencing labor (Figure 1, A and C) versus GA-matched decidua basalis and parietalis from women

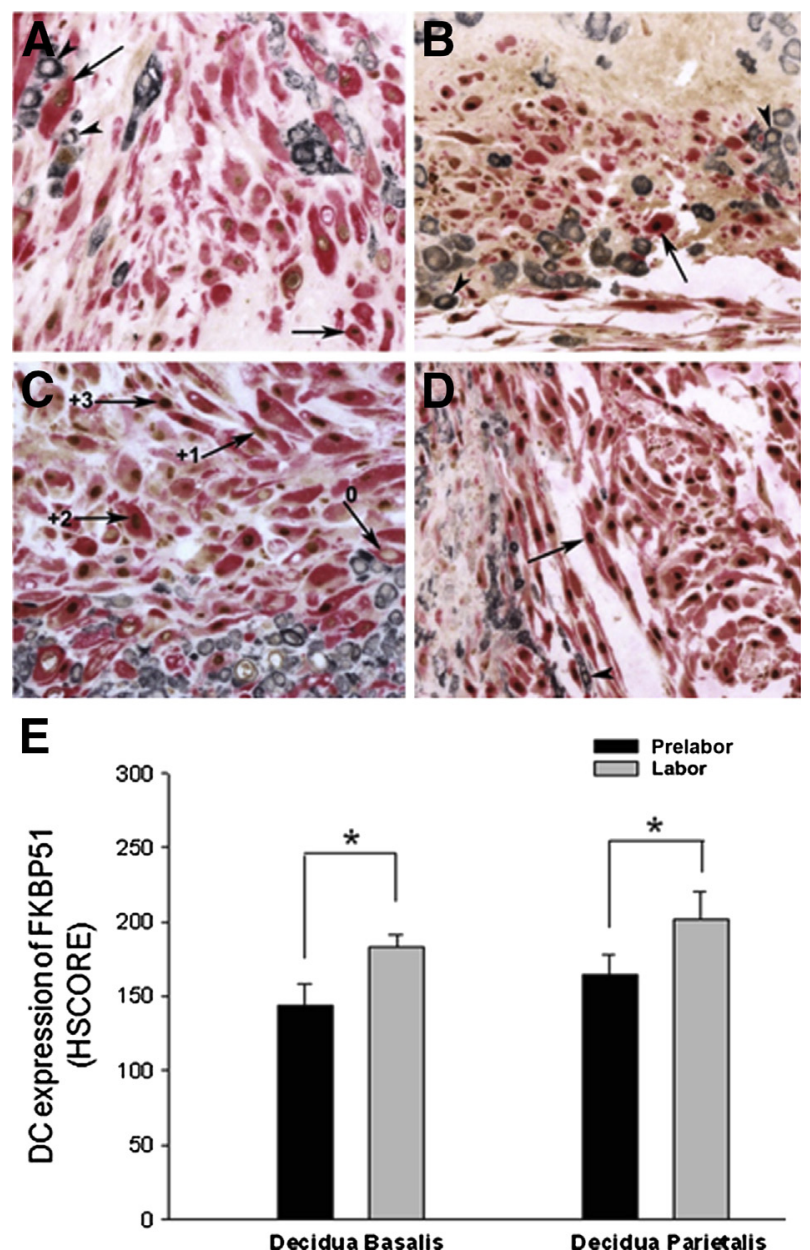

Figure 1 Immunoreactive FKBP51 in prelabor versus post-labor decidua. Decidual tissues were immunostained for FKBP51 (brown in nucleus) and vimentin (red in cytoplasm) to identify decidual cells (DCs; arrows) or cytokeratin (gray in cytoplasm) to identify interstitial trophoblasts (arrowheads). Representative FKBP51 staining is shown in decidua basalis (A) and parietalis (C) before labor and decidua basalis (B) and parietalis (D) during labor. In C, 0 indicates no staining intensity for FKBP51 immunoreactivity, +1 indicates weak staining intensity for FKBP51 immunoreactivity, +2 indicates moderate staining intensity for FKBP51 immunoreactivity, and +3 indicates strong staining intensity for FKBP51 immunoreactivity. E: HSCORE for FKBP51 expression. Data represent HSCORE means \pm SEM (E). $n=8$ (E, paired decidua basalis and parietalis from cesarean delivery); $n=9$ (E, paired decidua basalis and parietalis from vaginal delivery). ${ }^{*} P<0.05$.

after labor (Figure 1, B and D). Immunoreactive FKBP51 is present at varying intensity in DCs with significantly higher HSCOREs (Figure 1E) in DC nuclei of GA-matched postlabor versus prelabor placental sections $(P<0.05)$ (Figure 1, A-D).

To complement in situ observations indicating highly restricted FKBP51 expression in DCs, qPCR analyses of primary cultures of term DCs, CYTs, and SCTs incubated with vehicle (control) or Dex for 6 hours were analyzed for FKBP51 and FKBP52 mRNA expression (Figure 2). Incubation of term DCs with Dex enhanced FKBP51 mRNA levels by more than ninefold $(P<0.001)$ versus control 

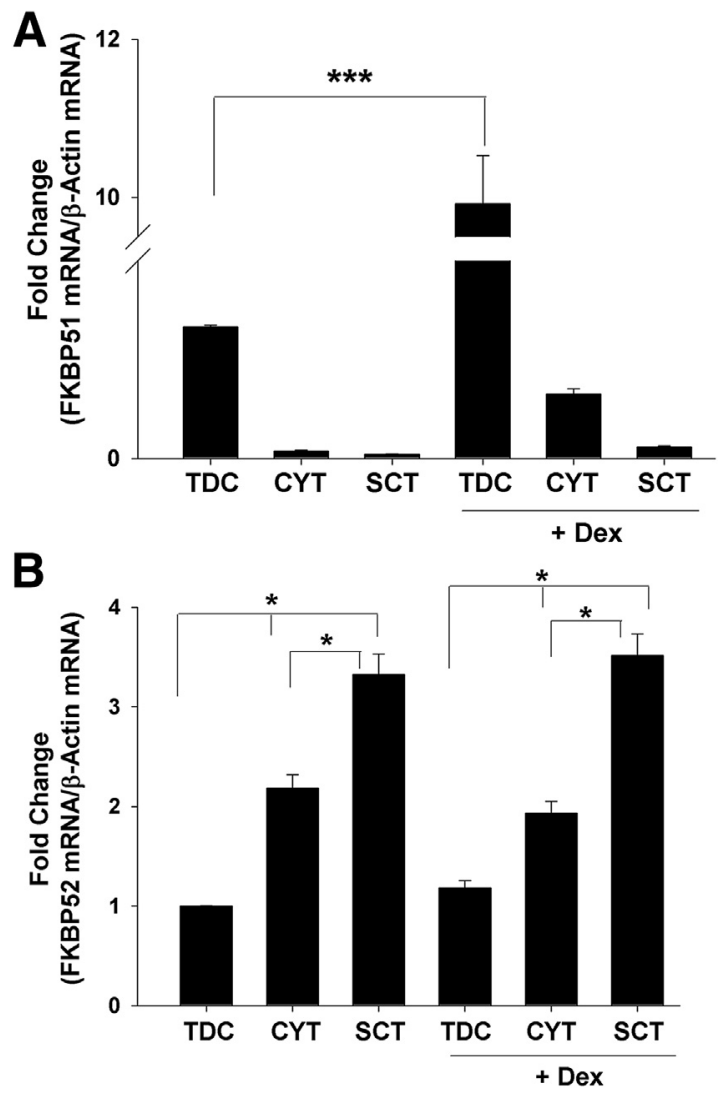

Figure 2 Comparison of FKBP51 and FKBP52 mRNA levels in term decidual cells (DCs), cytotrophoblasts (CYTs), and syncytiotrophoblasts (SCTs) by quantitative real-time PCR. FKBP51 (A) and FKBP52 (B) mRNA levels were determined and normalized to $\beta$-actin in term DCs (TDCS), CYTs, and SCTs after 6 hours of incubation with control (Cont) or $10^{-7} \mathrm{~mol} / \mathrm{L}$ Dex (+Dex). Data represent means \pm SEM from three replicates $(\mathbf{A}$ and $\mathbf{B}) . n=3$ (A and $\mathbf{B}) .{ }^{*} P<0.05,{ }^{* *} P<0.001$.

(Figure 2A). In contrast, although low levels of FKBP51 mRNA were present in CYT and SCT cultures, Dex elicited a similar fold increase in FKBP51 levels. However, this finding likely reflected DC contamination because qPCR for vimentin (an established DC marker) revealed presence of DCs in CYT and SCT cultures (data not shown). In contrast to the far greater expression of FKBP51 in term DCs compared with trophoblasts and the clear up-regulation of FKBP51 expression in term DCs by Dex, parallel experiments revealed higher FKBP52 mRNA levels in cultured CYTs and SCTs compared with term DCs, and a failure of Dex to affect FKBP52 mRNA expression in any of these cells (Figure 2B).

Progestin- or glucocorticoid-mediated regulation of steady-state mRNA levels for FKBP51 and FKBP52 in DC cultures were compared with $\mathrm{E}_{2}$ used as the control to mimic elevated estrogen levels during pregnancy (Figure 3). Compared with $\mathrm{E}_{2}$ alone, co-incubation with MPA or Dex significantly increased steady-state FKBP51 mRNA levels by 4.4- and 8.4-fold, respectively $(P<0.001)$ (Figure $3 \mathrm{~A}$ ). However, the increased response to $\operatorname{Org} 2058$ did not attain statistical significance, consistent with a predominantly glucocorticoid- rather than progestin-mediated regulation of FKBP51 gene expression. Conversely, versus $\mathrm{E}_{2}$ alone, coincubation with MPA, Dex, or Org2058 did not significantly alter low levels of steady-state FKBP52 mRNA levels (Figure 3B).

To investigate the direct effects of steroids in regulating FKBP51, FKBP52, PR, and GR protein expression in primary cultures of term DCs, experimental incubations were followed by immunoblotting of nuclear (Figure 4) and cytoplasmic (Figure 5) extracts. Representative immunoblotting (Figure 4A) and the accompanying graphs indicate the following: i) FKBP51 is strongly up-regulated by MPA and Dex and slightly increased by $\operatorname{Org} 2058$, whereas $E_{2}$ is ineffective when added alone or when added with each of the other steroids $(P<0.01)$ (Figure $4, \mathrm{~A}$ and $\mathrm{B})$; ii) FKBP52 is barely detected under any incubation condition (Figure 4A); and iii) both PR-A and PR-B isoforms are evident under all incubation conditions and are considered together in Figure 4C. Consistent with our previous report, ${ }^{10}$ incubation with $\mathrm{E}_{2}$ alone increased $\mathrm{PR}$ expression $(P<0.001)$ (Figure $4, \mathrm{~A}$ and $\mathrm{C})$. This $\mathrm{E}_{2}$-enhanced $\mathrm{PR}$ expression is significantly suppressed by co-incubation of
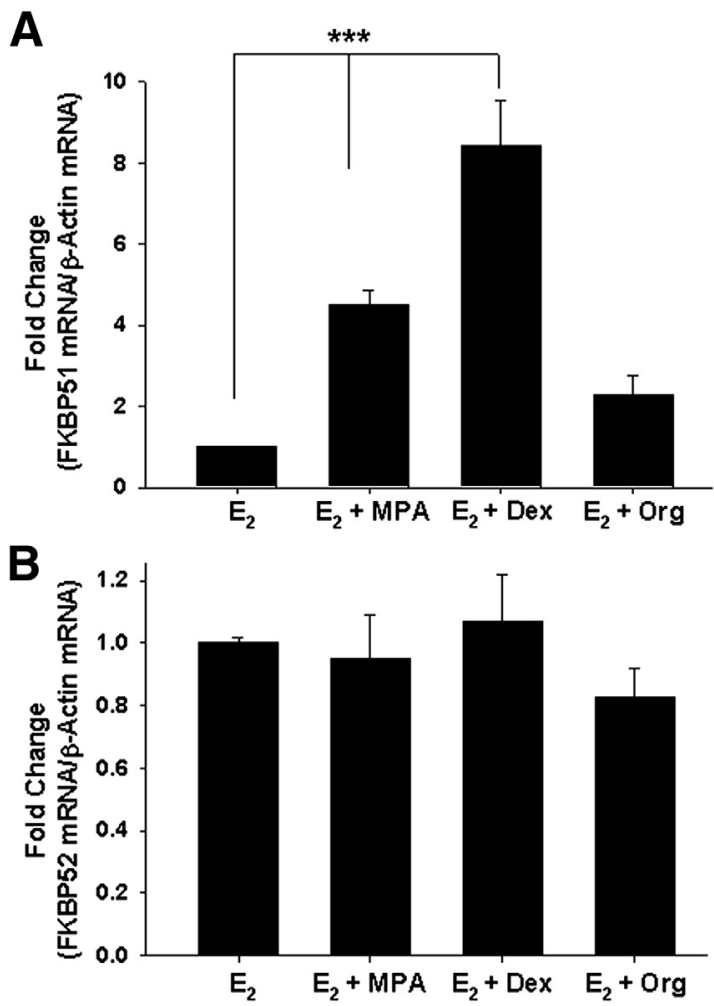

Figure 3 FKBP51 and FKBP52 mRNA expression in term decidual cell (DC) monolayers. Confluent leukocyte-free term DCs from decidua parietalis were primed for 7 days with $10^{-8} \mathrm{~mol} / \mathrm{L}$ estradiol $\left(E_{2}\right), E_{2}+10^{-7} \mathrm{~mol} / \mathrm{L}$ medroxyprogesterone acetate (MPA), $E_{2}+$ dexamethasone (Dex), or $E_{2}+$ Organon (Org) 2058, then incubated with corresponding steroids in DM for 6 hours. Quantitative real-time RT-PCR for FKBP51 (A) and FKBP52 (B) mRNA levels for term DCs measured and normalized to $\beta$-actin. Data represent means $\pm \operatorname{SEM}(\mathbf{A}$ and $\mathbf{B}) . n=4(\mathbf{A}$ and $\mathbf{B}) .{ }^{* * *} P<0.001$. DM, defined media. 

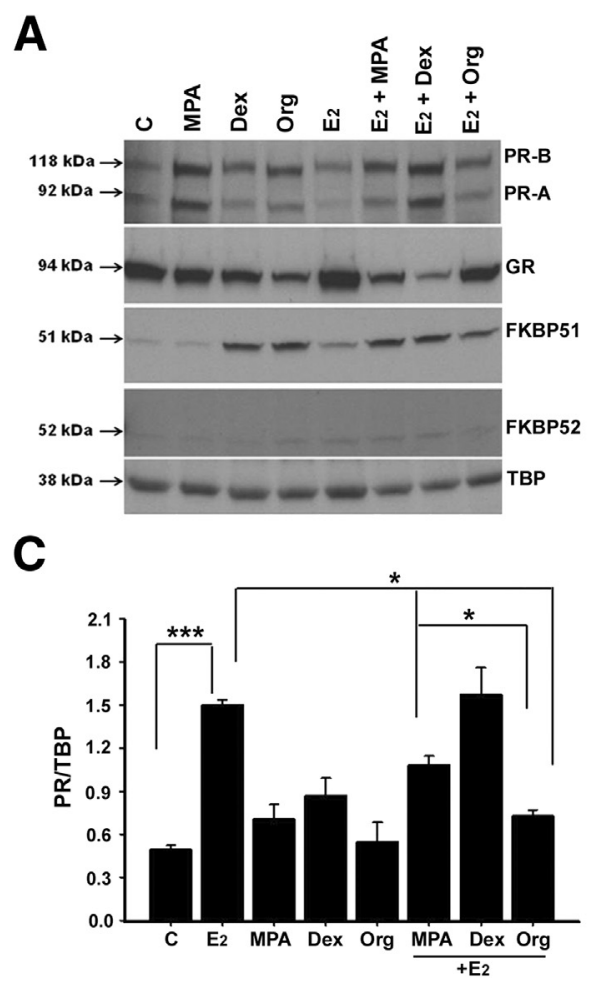

B

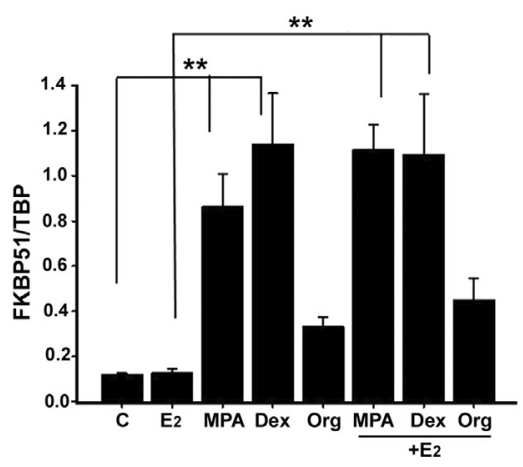

D

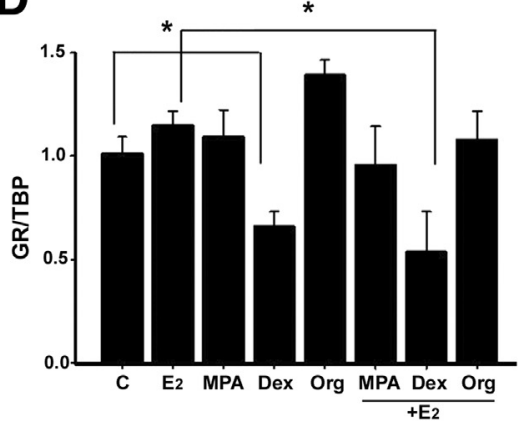

Figure 4 FKBP51, FKBP52, progesterone receptor (PR), and glucocorticoid receptor (GR) protein expression in nuclear extracts of decidual cell (DC) monolayers. Confluent leukocyte-free DCs were primed for 7 days with vehicle [control (C)], $10^{-8} \mathrm{~mol} / \mathrm{L}$ estradiol $\left(E_{2}\right), 10^{-7} \mathrm{~mol} / \mathrm{L}$ medroxyprogesterone acetate (MPA), $10^{-7} \mathrm{~mol} / \mathrm{L}$ dexamethasone (Dex), $10^{-7} \mathrm{~mol} / \mathrm{L}$ Organon (Org) 2058, $E_{2}+$ MPA, $E_{2}+$ Dex, or $E_{2}+$ Org2058, then incubated for 24 hours with corresponding steroids in DM. Immunoblot readings were normalized to TATA-binding protein (TBP) as loading control. A: Representative immunoblotting displaying bands for PR-A and PR-B isoforms, GR, FKBP51, FKBP52, and TBP. Graphs display immunoblot densitometry readings obtained from experimental incubations of term DC cultures for FKBP51/TBP (B), PR/TBP (C), and GR/TBP (D). Data represent means \pm SEM (C and D). $n=3$ (C and D). ${ }^{*} P<0.05$, $* * P<0.01$, and $* * * P<0.001$.
$\mathrm{E}_{2}$ with MPA or Org2058, but not Dex $(P<0.05)$ (Figure 4, $\mathrm{A}$ and $\mathrm{C}$ ); and iv) GR expression is significantly downregulated by incubation with Dex or co-incubation with $\mathrm{E}_{2}+$ Dex, but no significant changes in GR expression were evident in response to any other incubation conditions $(P<$ 0.05) (Figure 4, A and D).
Consistent with observations for nuclear extracts displayed in Figure 4, immunoblotting of the residual cytosol extracts (Figure 5) revealed parallel changes. Specifically, DCs exhibit an increase in FKBP51 expression in response to MPA or Dex $(P<0.05)$ (Figure 5, A and B), barely detectable FKBP52 expression under all incubation
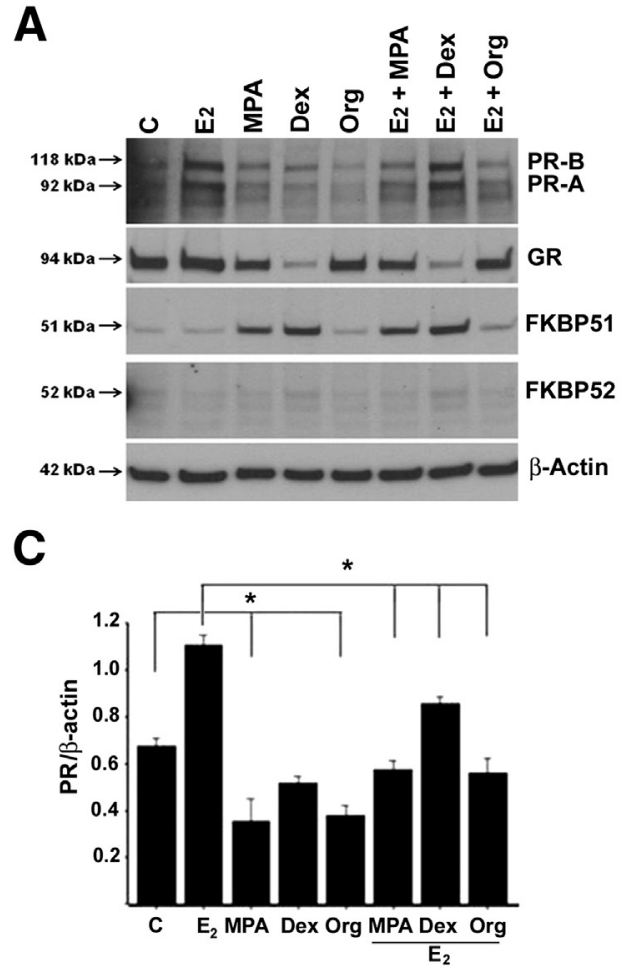

B
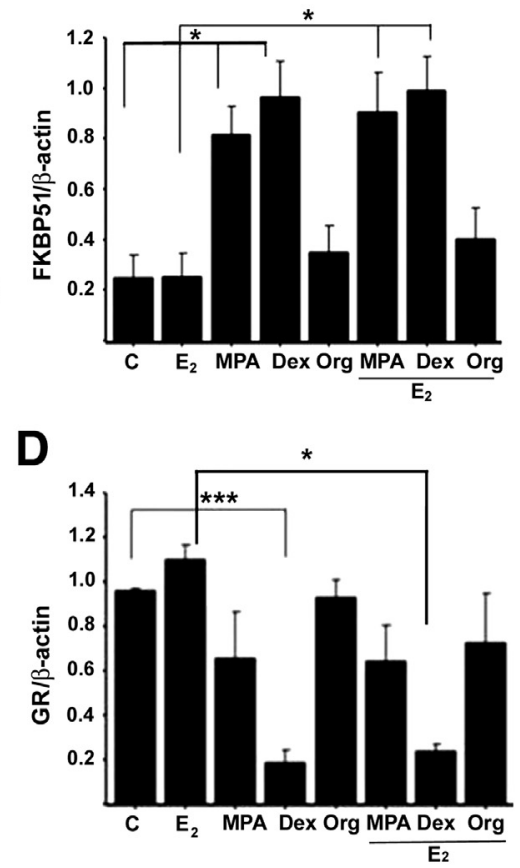

Figure 5 FKBP51, FKBP52, progesterone receptor (PR), and glucocorticoid receptor (GR) protein expression in cytosolic extracts of decidual cell (DC) monolayers. Cytosolic extracts from the experiments described in Figure 4 were analyzed by immunoblotting, and results were normalized to $\beta$-actin as loading control. A: Representative immunoblotting displaying bands for PR-A and $\mathrm{PR}-\mathrm{B}$ isoforms, GR, FKBP51, FKBP52, and $\beta$-actin. Graphs display densitometric analysis of immunoblot readings obtained from experimental incubations of term $D C$ cultures isolated from women for FKBP51/ $\beta$-actin (B), PR/ $\beta$-actin (C), and $\mathrm{GR} / \beta$-actin (D). Data represent means $\pm \mathrm{SEM}$ (B-D). $n=3$ (B-D). ${ }^{*} P<0.05,{ }^{* *} P<0.001$. 


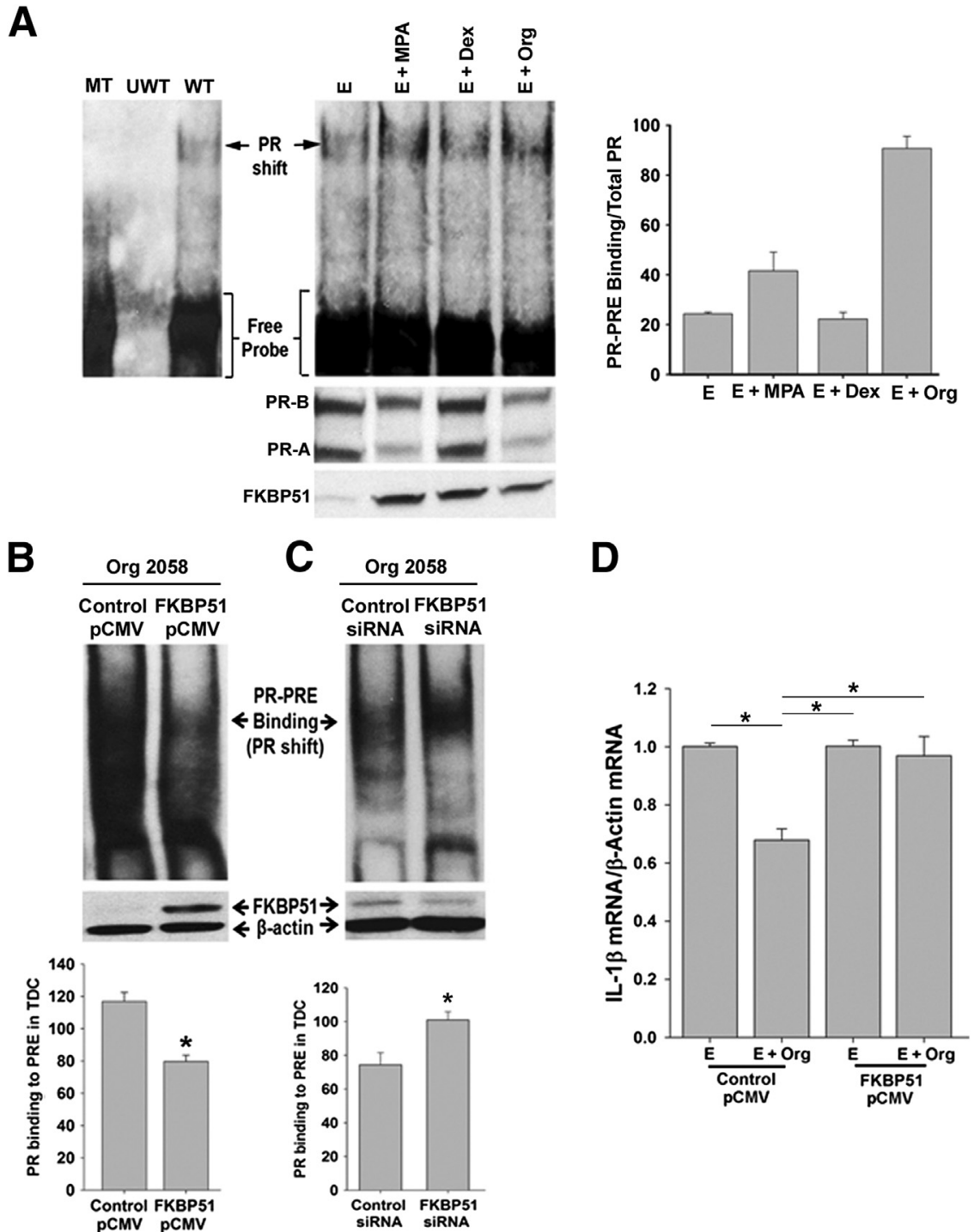

Figure 6 Role of FKBP51 in progesterone (PR) binding to DNA [progesterone response element (PRE)] by electrophoretic mobility shift assay (EMSA). A: In nuclear cell extracts obtained from term decidual cells (DCs), no PR shift occurs in labeled mutant PRE (MT) or unlabeled wild-type PRE (UWT), but a clear PR shift is seen labeled wild-type PRE (WT). EMSA displaying PR-PRE binding in DC nuclear extracts incubated with $10^{-8} \mathrm{~mol} / \mathrm{L}$ estradiol $\left(E_{2}\right), E_{2}+10^{-7} \mathrm{~mol} / \mathrm{L}$ medroxyprogesterone acetate (MPA), $E_{2}+10^{-7} \mathrm{~mol} / \mathrm{L}$ dexamethasone (Dex), or $\mathrm{E}_{2}+10^{-7} \mathrm{~mol} / \mathrm{L}$ Organon (Org) 2058. Respective immunoblot analyses for PR isoforms and FKBP51 in the decidual nuclear extracts from incubation described above. Graph displays ratio of PR-PRE binding/total PR level for each incubation condition. B: After confirming that FKBP51 is overexpressed in DCs after 48 hours of transfection with a FKBP51 vector (FKBP51 pCMV) or a control vector (Control pCMV) by immunoblotting; PR-PRE binding in control pCMV or FKBP51 pCMV-transfected DCs treated with $10^{-8} \mathrm{~mol} / \mathrm{L} \mathrm{E}_{2}+10^{-7} \mathrm{~mol} / \mathrm{L}$ Org2058 for 4 hours is detected by EMSA. Graph represents PR binding to PRE. After confirming silencing of FKBP51 in DCs at 48 hours after transfection with FKBP51 siRNA versus control siRNA by immunoblotting (C), PR-PRE binding in control or FKBP51 siRNAtransfected DCs treated with $10^{-8} \mathrm{~mol} / \mathrm{L} \mathrm{E}_{2}+10^{-7}$ $\mathrm{mol} / \mathrm{L}$ Org2058 for 4 hours detected by EMSA (B). Graph represents PR binding to PRE. D: IL-1 $\beta$ mRNA levels in control pCMV or FKBP51 pCMV-transfected term $D C$ cultures treated with $E_{2}$ versus $E_{2}+$ Org for 6 hours. Data represent means \pm SEM (A-D). $n=2$ (A); $n=3$ (B-D). ${ }^{*} P<0.05$.

conditions (Figure 5A), dominant down-regulation of PR-A and PR-B expression in co-incubations with $\mathrm{E}_{2}+\mathrm{MPA}$ or $\mathrm{E}_{2}+\operatorname{Org} 2058$ versus $\mathrm{E}_{2}$ alone $(P<0.05)$ (Figure 5, A and $C)$, and significantly down-regulated GR expression after incubation with Dex or co-incubation with $\mathrm{E}_{2}+$ Dex compared with control $(P<0.001)$ or $\mathrm{E}_{2}$ alone $(P<0.05)$, respectively (Figure 5, A and D).

To determine the functional impact of FKBP51 on PR, DNA (PRE) binding activity of PR was analyzed by EMSA in $E_{2}$ with or without progestin- or glucocorticoid-treated DC nuclear extracts (Figure 6). Incubation with $\mathrm{E}_{2}+\mathrm{MPA}$ or $\mathrm{E}_{2}+$ Org2058 induces greater PR-PRE binding versus $\mathrm{E}_{2}$ or $\mathrm{E}_{2}+$ Dex (Figure 6A). Moreover, after normalization to total PR levels, which are selectively reduced in the nuclear extracts by Org2058 compared with MPA (Figure 3C and Figure 6A), PR-PRE binding in incubations with $\mathrm{E}_{2}+$ Org2058 is higher than with $\mathrm{E}_{2}+$ MPA (Figure 6A). Thus, selective lowering of PR levels in response to $\mathrm{E}_{2}+$ the pure progestin, Org2058, is accompanied by greater PR-PRE binding, whereas less PR inhibition by the mixed glucocorticoid/progestin, MPA, is associated with lesser PR-PRE binding, reflecting concomitant up-regulation of FKBP51 by $\mathrm{E}_{2}+$ MPA (Figure 6A). Furthermore, FKBP51 overexpression in DCs lowers $\mathrm{E}_{2}+$ Org2058induced PR-PRE binding (Figure 6B), whereas silencing of FKBP51 enhances $E_{2}+$ Org2058-induced $\mathrm{PR}$ binding to PRE (Figure 6C). To confirm these EMSA results showing that reduced PR-PRE binding in FKBP51-overexpressed DCs modifies transcription of a PR-target gene, IL-1 $\beta$ mRNA expression in control and pCMV-FKBP51 vector-transfected term DCs treated with $\mathrm{E}_{2}$ or $\mathrm{E}_{2}+$ Org2058 were analyzed using qPCR. Compared with $\mathrm{E}_{2}, \mathrm{E}_{2}+$ Org2058 significantly inhibited basal mRNA expression of IL-1 $\beta$ (approximately $30 \% ; P<0.05)$ in control vector-transfected term DCs, and this inhibition of IL-1 $\beta$ mRNA expression was completely reversed in FKBP51-overexpressed DC cultures (Figure 6D). 


\section{Discussion}

\section{Labor-Related Regulation of FKBP51 Expression in Decidua}

Our prior study identified the cell type mediating functional P4 withdrawal at the human maternal-fetal interface by coordinating in situ with in vitro observations. ${ }^{10}$ In situ studies involved PR immunostaining in paired sections from GAmatched prelabor versus post-labor decidua parietalis and basalis. ${ }^{10}$ Because intermingling of morphologically similar DCs and ITs at the maternal-fetal interface makes differentiation of these two cell types difficult without specific molecular markers, we used sequential triple immunostaining to reveal that PR immunoreactivity is as follows: i) localized in nuclei of vimentin-positive DCs, ii) not detected in cytokeratin-positive ITs, and iii) significantly lower in DCs of GA-matched sections of post-labor versus prelabor decidua basalis and parietalis. ${ }^{10}$ Complementing these in situ observations, incubation of human term DCs with $\mathrm{E}_{2}$ markedly increased both PR-A and PR-B expression compared with steroid-free controls. Lower PR levels were observed in cultures incubated with MPA alone, and intermediate PR isoform levels resulted from incubations with $\mathrm{E}_{2}+\mathrm{MPA}^{10}$ These differential steroid effects are consistent with PR expression in endometrial stromal cells across the menstrual cycle, where PR expression is higher during the follicular versus luteal phase. ${ }^{29}$

The current study extends our previous report ${ }^{10}$ by demonstrating that the anti-progestin, FKBP51, is present in human decidua and acts as an additional mediator of functional P4 withdrawal. Specifically, immunostaining of GA-matched prelabor versus post-labor decidua basalis and parietalis sections indicates that like PR, FKBP51 is localized in DC nuclei but absent from adjacent ITs. However, unlike PR, term labor is accompanied by a significant increase in DC-expressed FKBP51 levels. These observations suggest the existence of a parturition-associated double hit in which enhanced FKBP51 levels in DCs inhibit the transcriptional activity of declining DC-expressed PR levels to amplify functional P4 withdrawal. On the other hand, previous studies have shown that increased expression of PR-A over PR-B isoform accompanies parturition in myometrial cells. ${ }^{8,9}$ In analysis of term DCs, both PR isoforms display parallel changes in response to steroid treatments, suggesting differential tissue- and/or cell-specific mechanisms of PR isoform regulation.

\section{Role of FKBP51 in Inducing Functional P4 Withdrawal during Labor}

Because the GR has also been implicated in mediating functional P4 withdrawal, ${ }^{11}$ we assessed GR expression in term decidua and demonstrated constitutively expressed GR in both DCs and ITs. ${ }^{10}$ Several reports indicate that progestin- and glucocorticoid-induced FKBP51 triggers a negative feedback loop inhibiting PR- and GR-mediated transcription. $^{30,31}$ Thus, we incubated primary cultures of human DCs with the synthetic steroids: MPA, a mixed progestin-glucocorticoid agonist; Dex, a classic glucocorticoid $^{19}$; and Org2058, a pure progestin agonist. ${ }^{20}$ Then, we assessed FKBP51, FKBP52, PR, and GR expression. Immunoblotting indicated that, as reported in other cell types, ${ }^{32,33}$ FKBP51 is present in the nucleus and cytoplasm of cultured DCs and that glucocorticoid pays a dominant role in reciprocally up-regulating FKBP51 and down-regulating GR expression. By comparison with FKBP51, PR expression displayed dominant progestin-mediated down-regulation. In contrast with highly regulated FKBP51 expression, FKBP52 is essentially unregulated by either progestin or glucocorticoid. Measurements of steady-state mRNA levels are consistent with changes in FKBP51 protein expression.

Stress induces the hypothalamic pituitary axis to release corticotropin-releasing hormone and adrenocorticotropic hormone, which elicit cortisol secretion from adrenal glands to activate GR in several tissues. In turn, GR activation ultimately generates a negative feedback loop by inhibiting corticotropin-releasing hormone and adrenocorticotropic hormone release, which enables the body to tolerate acute stress responses. Recent studies reveal that FKBP51 levels, increased by glucocorticoids, impair GR function to generate an ultrashort negative feedback loop. ${ }^{34}$ Several research groups demonstrated that FKBP51 knockout or knockdown exerts a protective effect in animal models of depression and anxiety. ${ }^{35}$ Thus, we posit that maturation of the fetal hypothalamic pituitary axis and/or increasing maternal glucocorticoid levels trigger parturition-associated increases in DC FKBP51 expression. Strong evidence now links maternal stress and depression to preterm birth. ${ }^{36}$ Moreover, although glucocorticoids are routinely administered to promote fetal lung maturity, ${ }^{37}$ repeated doses may shorten the duration of pregnancy, ${ }^{38}$ and chronic glucocorticoid therapy is associated with preterm birth. ${ }^{39}$ These links among stress, glucocorticoid treatment, and prematurity, and the emergence of FKBP51 as a promising new target for stress-related disorders, ${ }^{35}$ suggest stress-induced FKBP51 as a crucial mediator of preterm birth.

Consistent with these hypotheses, treatment of cultured DCs with Dex, a pure glucocorticoid, or MPA, a mixed progestin/glucocorticoid, resulted in significant upregulation of DC FKBP51 mRNA and protein expression. This suggests that increasing fetal, placental, and/or maternal glucocorticoid levels before both term and preterm birth trigger functional P4 withdrawal via increased DC FKBP51 expression. The current study provides support for this proposed mechanism by demonstrating that overexpression of FKBP51 in DC cultures inhibits PR binding to its canonical DNA response element, consistent with reduced PR-mediated transcriptional activity. Conversely, PR binding to PRE is increased in DCs in which FKBP51 has been silenced. Furthermore, lack of inhibitory effect of pure progestin Org2058 on the IL-1 $\beta$ transcript in FKBP51 overexpression DCs further supports our hypothesis that FKBP51 inhibits transcriptional activity of PR in DCs. 
Extrapolation of these in vitro results to the in situ milieu accompanying labor suggests that glucocorticoid-enhanced DC expression of FKBP51 is a strong contributor to labor-related functional P4 withdrawal by inhibiting transcriptional activity of declining PR levels.

Unlike our observations with DCs, immunostaining failed to detect FKBP51 in ITs of term decidua. This apparent absence of FKBP51 expression in situ and its low glucocorticoid responsiveness in vitro is particularly noteworthy in view of the tight negative feedback that normally exists between expression of FKBP51 and GR activity. ${ }^{31,34}$ Thus, the absence of FKBP51 in ITs suggests the existence of a previously undisclosed phenomenon of unopposed GR expression in trophoblasts, which may be relevant to parturition. For example, glucocorticoid induction of $11 \beta$ hydroxysteroid dehydrogenase type 1 expression in human chorionic trophoblasts ${ }^{40}$ supports a paracrine mechanism whereby adjacent ITs enhance local glucocorticoid levels in adjacent DCs via conversion of cortisone to cortisol to promote parturition.

\section{Supplemental Data}

Supplemental material for this article can be found at http://dx.doi.org/10.1016/j.ajpath.2015.05.014.

\section{References}

1. Kastner P, Krust A, Turcotte B, Stropp U, Tora L, Gronemeyer H, Chambon P: Two distinct estrogen-regulated promoters generate transcripts encoding the two functionally different human progesterone receptor forms A and B. EMBO J 1990, 9:1603-1614

2. Thijssen JH: Progesterone receptors in the human uterus and their possible role in parturition. J Steroid Biochem Mol Biol 2005, 97: 397-400

3. Zakar T, Hertelendy F: Progesterone withdrawal: key to parturition. Am J Obstet Gynecol 2007, 196:289-296

4. Block BS, Liggins GC, Creasy RK: Preterm delivery is not predicted by serial plasma estradiol or progesterone concentration measurements. Am J Obstet Gynecol 1984, 150:716-722

5. Palliser HK, Zakar T, Symonds IM, Hirst JJ: Progesterone receptor isoform expression in the guinea pig myometrium from normal and growth restricted pregnancies. Reprod Sci 2010, 17:776-782

6. Neilson JP: Mifepristone for induction of labour. Cochrane Database Syst Rev 2000, (4):CD002865

7. Astle S, Slater DM, Thornton S: The involvement of progesterone in the onset of human labour. Eur J Obstet Gynecol Reprod Biol 2003, 108:177-181

8. Merlino AA, Welsh TN, Tan H, Yi LJ, Cannon V, Mercer BM, Mesiano S: Nuclear progesterone receptors in the human pregnancy myometrium: evidence that parturition involves functional progesterone withdrawal mediated by increased expression of progesterone receptor-A. J Clin Endocrinol Metab 2007, 92:1927-1933

9. Mesiano S, Chan EC, Fitter JT, Kwek K, Yeo G, Smith R: Progesterone withdrawal and estrogen activation in human parturition are coordinated by progesterone receptor A expression in the myometrium. J Clin Endocrinol Metab 2002, 87:2924-2930

10. Lockwood CJ, Stocco C, Murk W, Kayisli UA, Funai EF, Schatz F: Human labor is associated with reduced decidual cell expression of progesterone, but not glucocorticoid, receptors. Am J Obstet Gynecol 2010, 95:2271-2275

11. Karalis K, Goodwin G, Majzoub JA: Cortisol blockade of progesterone: a possible molecular mechanism involved in the initiation of human labor. Nat Med 1996, 2:556-560

12. Bigsby RM, Young PC: Progesterone and dexamethasone inhibition of uterine epithelial cell proliferation: studies with antiprogesterone compounds in the neonatal mouse. J Steroid Biochem Mol Biol 1993, $46: 253-257$

13. Sun Y, Fang M, Davies H, Hu Z: Mifepristone: a potential clinical agent based on its anti-progesterone and anti-glucocorticoid properties. Gynecol Endocrinol 2013, 30:169-173

14. Tranguch S, Smith DF, Dey SK: Progesterone receptor requires a cochaperone for signalling in uterine biology and implantation. Reprod Biomed Online 2007, 14 Spec No 1:39-48

15. Ratajczak T, Ward BK, Minchin RF: Immunophilin chaperones in steroid receptor signalling. Curr Top Med Chem 2003, 3:1348-1357

16. Sanchez ER: Chaperoning steroidal physiology: lessons from mouse genetic models of Hsp90 and its cochaperones. Biochim Biophys Acta 2012, 1823:722-729

17. Riggs DL, Cox MB, Cheung-Flynn J, Prapapanich V, Carrigan PE Smith DF: Functional specificity of co-chaperone interactions with Hsp90 client proteins. Crit Rev Biochem Mol Biol 2004, 39: 279-295

18. Jaaskelainen T, Makkonen H, Palvimo JJ: Steroid up-regulation of FKBP51 and its role in hormone signaling. Curr Opin Pharmacol 2011, $11: 326-331$

19. Africander D, Verhoog N, Hapgood JP: Molecular mechanisms of steroid receptor-mediated actions by synthetic progestins used in HRT and contraception. Steroids 2011, 76:636-652

20. Petit E, Courtin A, Kloosterboer HJ, Rostene W, Forgez P, Gompel A: Progestins induce catalase activities in breast cancer cells through PRB isoform: correlation with cell growth inhibition. J Steroid Biochem Mol Biol 2009, 115:153-160

21. Romero R, Yoon BH, Mazor M, Gomez R, Diamond MP, Kenney JS, Ramirez M, Fidel PL, Sorokin Y, Cotton D, Sehgal P: The diagnostic and prognostic value of amniotic fluid white blood cell count, glucose, interleukin-6, and gram stain in patients with preterm labor and intact membranes. Am J Obstet Gynecol 1993, 169:805-816

22. Lockwood CJ, Arcuri F, Toti P, Felice CD, Krikun G, Guller S, Buchwalder LF, Schatz F: Tumor necrosis factor-alpha and interleukin-1beta regulate interleukin- 8 expression in third trimester decidual cells: implications for the genesis of chorioamnionitis. Am J Pathol 2006, 169:1294-1302

23. Lockwood CJ, Murk W, Kayisli UA, Buchwalder LF, Huang ST, Funai EF, Krikun G, Schatz F: Progestin and thrombin regulate tissue factor expression in human term decidual cells. J Clin Endocrinol Metab 2009, 94:2164-2170

24. Tang Z, Tadesse S, Norwitz E, Mor G, Abrahams VM, Guller S: Isolation of hofbauer cells from human term placentas with high yield and purity. Am J Reprod Immunol 2011, 66:336-348

25. Lee MJ, Wang Z, Yee H, Ma Y, Swenson N, Yang L, Kadner SS, Baergen RN, Logan SK, Garabedian MJ, Guller S: Expression and regulation of glucocorticoid receptor in human placental villous fibroblasts. Endocrinology 2005, 146:4619-4626

26. Murk W, Atabekoglu CS, Cakmak H, Heper A, Ensari A, Kayisli UA, Arici A: Extracellularly signal-regulated kinase activity in the human endometrium: possible roles in the pathogenesis of endometriosis. J Clin Endocrinol Metab 2008, 93:3532-3540

27. Guzeloglu-Kayisli O, Halis G, Taskiran S, Kayisli UA, Arici A: DNAbinding ability of NF-kappaB is affected differently by ERalpha and ERbeta and its activation results in inhibition of estrogen responsiveness. Reprod Sci 2008, 15:493-505

28. Bradford MM: A rapid and sensitive method for the quantitation of microgram quantities of protein utilizing the principle of protein-dye binding. Anal Biochem 1976, 72:248-254 
29. Eckert RL, Katzenellenbogen BS: Human endometrial cells in primary tissue culture: modulation of the progesterone receptor level by natural and synthetic estrogens in vitro. J Clin Endocrinol Metab 1981, 52:699-708

30. Hubler TR, Denny WB, Valentine DL, Cheung-Flynn J, Smith DF, Scammell JG: The FK506-binding immunophilin FKBP51 is transcriptionally regulated by progestin and attenuates progestin responsiveness. Endocrinology 2003, 144:2380-2387

31. Stechschulte LA, Sanchez ER: FKBP51: a selective modulator of glucocorticoid and androgen sensitivity. Curr Opin Pharmacol 2011, $11: 332-337$

32. Tatro ET, Everall IP, Kaul M, Achim CL: Modulation of glucocorticoid receptor nuclear translocation in neurons by immunophilins FKBP51 and FKBP52: implications for major depressive disorder. Brain Res 2009, 1286:1-12

33. Toneatto J, Guber S, Charo NL, Susperreguy S, Schwartz J, Galigniana MD, Piwien-Pilipuk G: Dynamic mitochondrial-nuclear redistribution of the immunophilin FKBP51 is regulated by the PKA signaling pathway to control gene expression during adipocyte differentiation. J Cell Sci 2013, 126:5357-5368

34. Storer CL, Dickey CA, Galigniana MD, Rein T, Cox MB: FKBP51 and FKBP52 in signaling and disease. Trends Endocrinol Metab 2011, $22: 481-490$
35. Schmidt MV, Paez-Pereda M, Holsboer F, Hausch F: The prospect of FKBP51 as a drug target. ChemMedChem 2012, 7:1351-1359

36. Yonkers KA, Norwitz ER, Smith MV, Lockwood CJ, Gotman N, Luchansky E, Lin H, Belanger K: Depression and serotonin reuptake inhibitor treatment as risk factors for preterm birth. Epidemiology 2012, 23:677-685

37. McKinlay CJ, Crowther CA, Middleton P, Harding JE: Repeat antenatal glucocorticoids for women at risk of preterm birth: a Cochrane Systematic Review. Am J Obstet Gynecol 2012, 206:187-194

38. Guinn DA, Atkinson MW, Sullivan L, Lee M, MacGregor S, Parilla BV, Davies J, Hanlon-Lundberg K, Simpson L, Stone J, Wing D, Ogasawara K, Muraskas J: Single vs weekly courses of antenatal corticosteroids for women at risk of preterm delivery: a randomized controlled trial. JAMA 2001, 286:1581-1587

39. Silver RK, MacGregor SN, Sholl JS, Hobart JM, Neerhof MG, Ragin A: Comparative trial of prednisone plus aspirin versus aspirin alone in the treatment of anticardiolipin antibody-positive obstetric patients. Am J Obstet Gynecol 1993, 169:1411-1417

40. Li W, Gao L, Wang Y, Duan T, Myatt L, Sun K: Enhancement of cortisol-induced 11 beta-hydroxysteroid dehydrogenase type 1 expression by interleukin 1beta in cultured human chorionic trophoblast cells. Endocrinology 2006, 147:2490-2495 